\title{
Research Flights on Blue Origin's New Shepard
}

Erika B. Wagner

Blue Origin, 21218 76th Ave S, Kent, Washington, USA 98032

Abstract

Blue Origin's New Shepard launch vehicle made its first flight above the Kármán Line, returning safely to Earth in November 2015. At the time when this paper is being written (February 2021), the system has conducted 14 flights, including 10 with research and education payloads aboard. More than 100 payloads have exercised a wide range of capabilities and interfaces, from small cubesat-form factor student payloads to large custom payloads of nearly $100 \mathrm{~kg}$. Investigations have spanned a wide range of high-altitude and microgravity research objectives, as well as raising technology readiness level (TRL) on diverse hardware. This paper summarizes New Shepard's payload missions to date, and presents standardized and custom accommodations, both in the shirtsleeve cabin and directly exposed to the space environment.

Keywords

Microgravity $\cdot$ Suborbital $\cdot$ Technology $・$ Education

\section{INTRODUCTION}

Blue Origin's New Shepard system is a suborbital vehicle designed to carry up to six astronauts and/or payload stacks to space above the Kármán Line $(328,000 \mathrm{ft}, 100 \mathrm{~km})$. It consists of a booster rocket and a separable Crew Capsule. The stacked vehicle is approximately $60 \mathrm{ft}(18 \mathrm{~m})$ in height and $10-12 \mathrm{ft}(3-3.6 \mathrm{~m})$ in diameter. It is powered by a single $110,000-$ lbf $(489,304 \mathrm{~N})$ thrust BE-3 liquid rocket engine.

The $530 \mathrm{cu}-\mathrm{ft}$ (15 cu-m) capsule interior is designed to accommodate up to six astronaut seats or payload stacks in a circular configuration. Each payload stack can in turn support up to six Single Payload Lockers, or an equivalent combination of Single and Double Lockers. Additional accommodations have been developed to support sensors and antennae adjacent to windows, larger custom payloads, smaller educational payloads, and a wide range of other customer needs. Space-exposed payload accommodations are also available on the booster to support aspects of research which need direct access to the space environment or unencumbered views of the Earth and space.

After liftoff, the vehicle ascends vertically for approximately $150 \mathrm{~s}$ before main engine cutoff in the upper atmosphere. Several seconds later, the capsule separates from the booster and is pushed away by mechanical springs. From this point, the capsule and booster coast and reenter separately, as shown in Figure 1. The vehicles experience microgravity for a period of about 3 min before returning to Earth. The booster maneuvers back to a landing pad, restarts its engine, and deploys landing gear to perform a rocket-powered vertical landing. The capsule reenters and lands under a three-parachute canopy with the assistance of a retro-thrust system to reduce landing loads. The vehicles are then recovered and prepared for reflight.

On November 23, 2015, the New Shepard system made history by becoming the first rocket to ascend above the Kármán Line $(100 \mathrm{~km})$ and successfully return to Earth for a vertical landing. Less than 2 months later, on January 22, 2016, the same booster flew again, demonstrating reuse. Over the course of New Shepard's 14 test flights to date, the system has reached a number of other significant milestones, including the following:

- A record-setting booster that has flown to space and back seven times;

- $\quad$ Standard mission apogees up to 351,000 ft (66 mi, $107 \mathrm{~km}$ );

- Multiple successful tests of the full-envelope capsule escape systems;

- Ten missions with research and education payloads onboard, including the NS-11 flight on May 2, 2019, which carried 38 payloads.

\section{PAYLOAD CAPABILITIES}

The primary Payload capabilities of the New Shepard vehicle include the following:

${ }^{\dagger}$ Corresponding author: Erika B. Wagner

E-mail: ewagner@blueorigin.com 


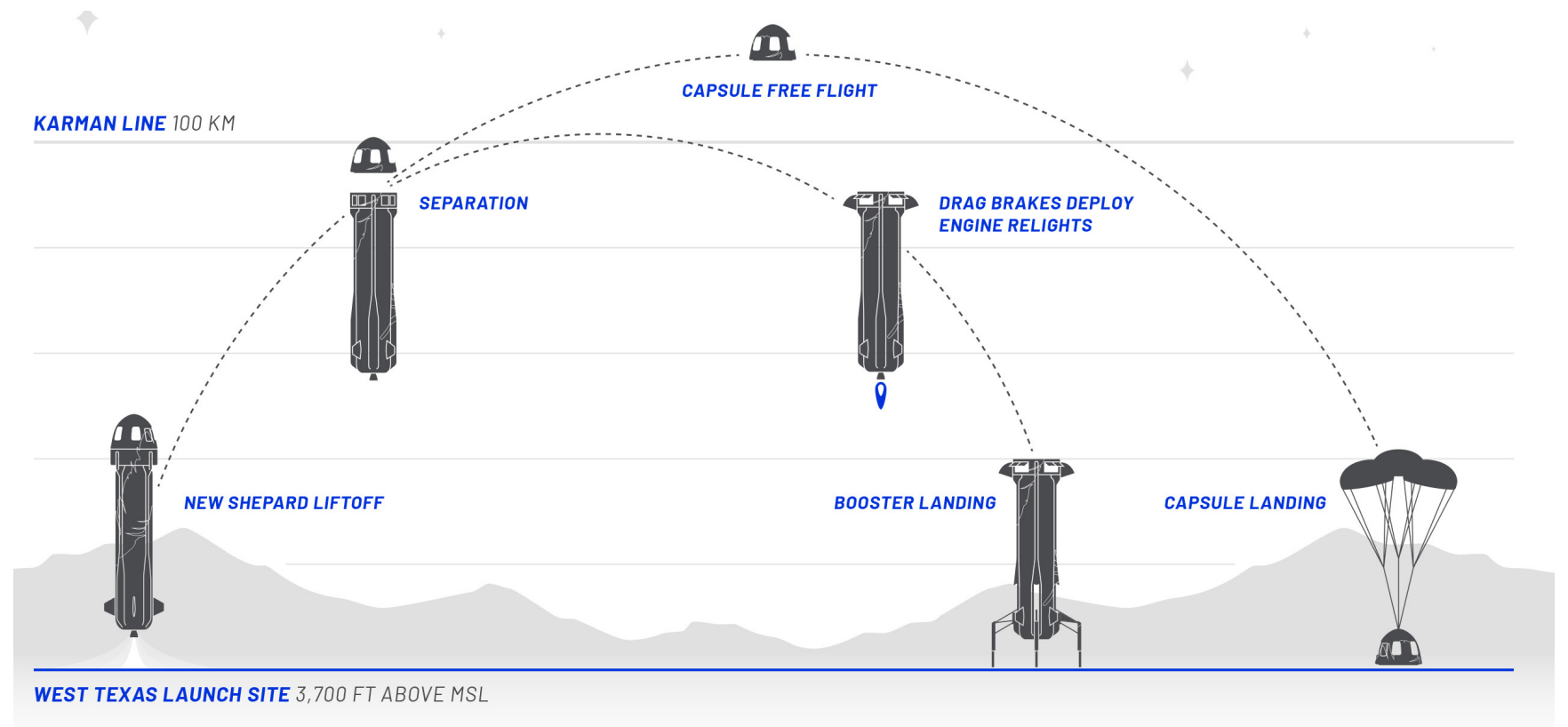

Figure 1. New Shepard flight profile, showing separation of the booster and capsule elements with independent return from above the Kármán Line.

- The ability to launch payloads, and ultimately payload operators, to suborbital altitudes on a frequent basis;

- Flights to altitudes above the Kármán Line $(100 \mathrm{~km}$, 62 miles), providing approximately $3 \mathrm{~min}$ in a high-quality microgravity environment;

- Recovery of payloads to facilitate sample analysis and system reflights;

- Availability of a high-volume cabin with shirt-sleeve environments, that is able to accommodate multiple experiments and, ultimately, researchers;

- Standard interfaces to mount experiments and provide power, cooling, command and control, and video and data recording;

- Rapid post-landing access by ground personnel to timesensitive payloads;

- Short timelines from contracting to flight-of the order of months rather than years-enabling high throughput research for academic, industrial, and educational users.

\section{PAYLOAD HISTORY}

New Shepard's ten payload flights have carried numerous research and education payloads for a variety of schools, universities, government agencies, and private companies.

Payloads to date have spanned a wide range of use cases, including the following:

- Basic and applied microgravity sciences;

- Earth and space sciences;
- Technology development;

- Education and public outreach;

- Marketing and market research.

Key research arenas and a sampling of payloads flown on the platform to date are included below.

Fluid physics studies have been the most prevalent area of academic research pursuit on New Shepard. These payloads can take advantage of longer equilibrium times than parabolic flights allow, supporting larger fluid volumes, as well as fluid transfers and complex multiphase flows.

- Modal Propellant Gauging, Carthage College Space Sciences Program with NASA Kennedy Space Center Cryogenics Laboratory: The experiment led by Dr. Kevin Crosby demonstrated a way to measure fuel levels in microgravity by using sound waves.

- Zero-Gravity Green Propellant Management Technology, Purdue University: Dr. Steven Collicott's payload aimed to help advance the use of a safer and more environmentally friendly rocket propellant by better understanding the fuel's behavior in microgravity.

Low-G collisions at multiple physical scales can model planetary science phenomena, including behavior in protoplanetary disks and surface interactions on asteroids.

- GAGa (Granular Anisotropic Gases), Otto-vonGuericke University, Magdeburg, Germany with OLYMPIASPACE, Darmstadt, Germany: With funding from the German space agency, DLR, the GAGa payload 
investigated the statistics of granular gases, which are dilute collections of solid grains that interact by random collisions. Data from GAGa will help validate existing theoretical models and contribute to understanding the dynamics of related systems such as avalanches and cosmic dust clouds.

Acute biological responses can be observed in short-time constant systems, as well as providing a unique opportunity to study the immediate response to G-transitions in a variety of cells, tissues, and model organisms, an area traditionally obscured by orbital mission timelines.

- Validating Telemetric Imaging Hardware for CrewAssisted and Crew-Autonomous Biological Imaging in Suborbital Applications, University of Florida, Gainesville, Florida, USA: Dr. Rob Ferl and Dr. AnnaLisa Paul have adapted technology designed for imaging plant growth and thermal responses on the ISS to suborbital uses. By recalibrating the way in which data are collected, the experiment will enable more biological research to be conducted on suborbital missions.

Technology demonstrations are particularly well-suited to the range of technology readiness levels (TRL) between 4 and 8 , showing behavior in relevant space environments, including launch and reentry survival, as well as microgravity behavior of G-sensitive systems like deployable structures and multiphase flows. This can provide risk reduction for future work on satellites, the International Space Station, or other destinations.

- Flow Boiling in Microgap Coolers, NASA Goddard Space Flight Center, Greenbelt, Maryland, USA: Cooling tightly packed electronics onboard a spacecraft can be challenging, and many solutions have not been able to undergo robust testing. Principal Investigator Franklin Robinson tested one of these solutions in a series of flights on New Shepard.

- Evolved Medical Microgravity Suction Device, Orbital Medicine, Richmond, Virginia, USA with Purdue University, West Lafayette, Indiana, USA: Orbital Medicine, a small business focused on aerospace medicine, received funding from NASA's Flight Opportunities Program to demonstrate an experimental medical technology designed to treat a collapsed lung in zero gravity (traditionally a gravity-dependent procedure). Over two flights, the team was able to evolve a phaseseparation technology that could potentially save lives in space.

Art and design payloads use a combination of launch, microgravity, and reentry and environments to explore novel artistic practices and design spaces.
- MIT Media Lab Space Exploration Initiative, Cambridge, Massachusetts, USA: The Space Exploration Initiative unites artists, engineers, scientists, and designers. Telepresent Drawings in Space used graphite to create a drawing that could only have been made in space. Living Distance: A Spider-Inspired Robotic Dance in Weightlessness demonstrated a crystalline robotic device that navigated zero gravity, similar to a performance. Other payloads from this team included TESSERAE: Self Assembling Space Architecture, Floral Cosmonauts: Crystal Electro-Nucleation and Queen Bee Maiden Flight.

Educational payloads offer students of all ages an opportunity to develop spaceflight systems and conduct unique experiments outside the bounds of their daily experience. By involving students in not only the science and technology of the payload, but also the marketing and communication, educators can engage their whole community.

- DCS Montessori Middle School, Castle Pines, Colorado, USA with DreamUp: This payload was a collaboration across nearly 500 K-8 students. An Arduino Nano microcontroller with a sensor package was designed and programed by the students to learn more about the environment inside the Crew Capsule. Further, a school-wide art project engaged all DCS Montessori students in creating novel pieces of personal expression.

Additional areas that are well suited to suborbital demonstration include the following:

Materials science investigations can take advantage of the absence of convection, sedimentation, and buoyancy effects to explore the production and interaction of novel materials, colloidal suspensions, crystallization, and solidification.

Combustion researchers can explore the physics of flame initiation, propagation, and quenching across a variety of fuels and substrates, including the unique microgravity properties of "cool flames." Fire safety demos can advance the state of technology.

Partial gravity missions are possible, using the spacecraft thrusters to spin the full capsule up to a stable rate of rotation to provide minutes of continuous access to centripetal accelerations between $0 \mathrm{G}$ and $1 \mathrm{G}$. With a $1.5 \mathrm{~m}$ radius, $11 \mathrm{rpm}$ rotation would support lunar-G explorations ranging from cryogenic fluid management and regolith management, to combustion and biological mechanotransduction.

Remote sensing payloads can take advantage of six windows with over $1,000 \mathrm{sq}-\mathrm{in}(6,400 \mathrm{sq}-\mathrm{cm})$ of viewable area each, allowing viewing of both Earth and space targets. Custom transparencies and externally mounted sensors can also support instruments measuring outside the visible spectrum. 


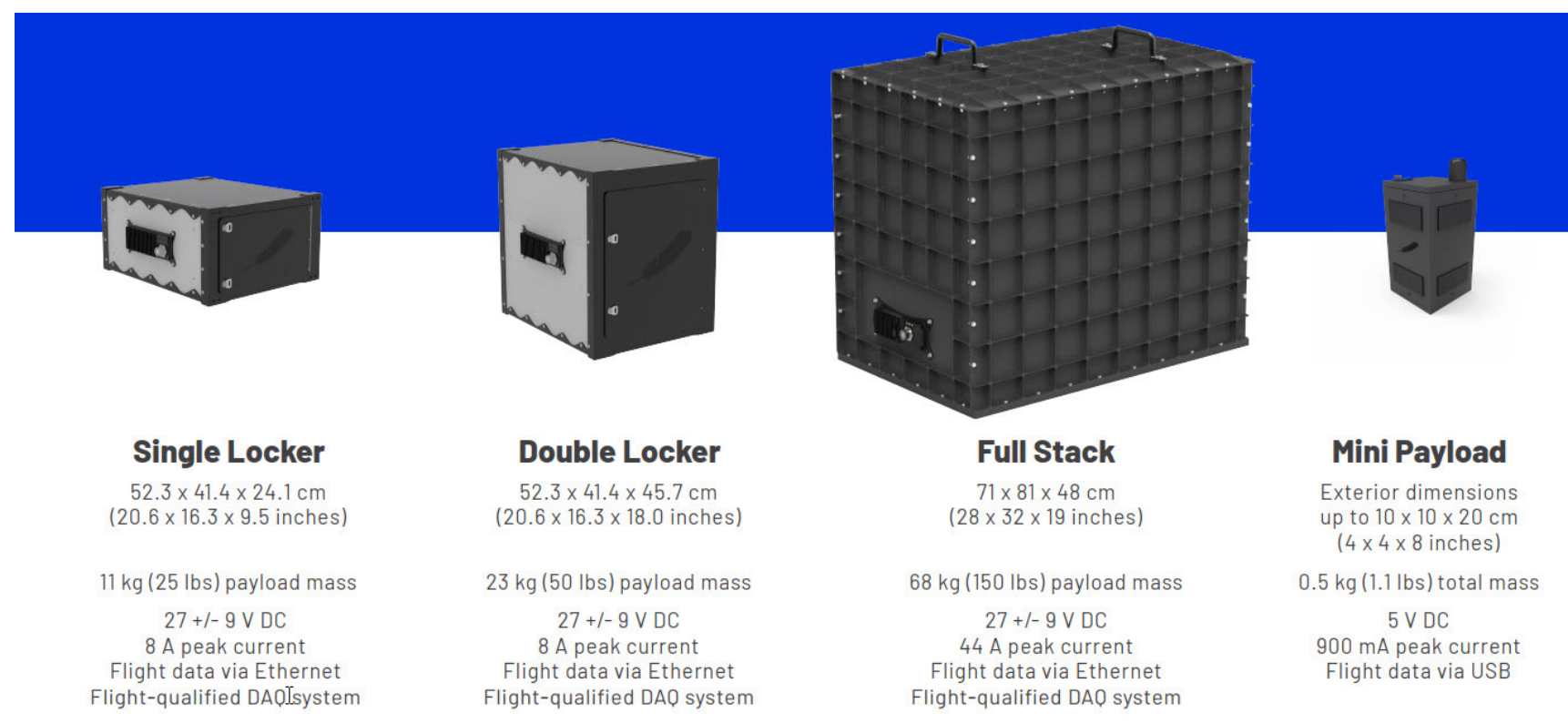

Figure 2. Four standardized interface options, with flight-qualified lockers and payload controllers provided for pre-flight testing, simplify mission readiness.

High altitude exposure of external payloads can support atmospheric measurement and sampling in the rarely accessed "ignorosphere" region between 37 and $66.5 \mathrm{mi}(60$ and $107 \mathrm{~km}$ ), exposure to hard vacuum and atomic oxygen, and remote sensing that is approximately three times higher than stratospheric balloons.

Future human-tended payload missions will also support investigations into acute biomedical responses to spaceflight with a large, diverse population of non-traditional spaceflight participants. They will also enable expert users to support direct hands-on payload operation, rather than relying on automation or astronaut training.

\section{PAYLOAD INTERFACES}

Interfaces, environments, and operations for payloads flown in the New Shepard capsule are described in detail in the New Shepard Payload User's Guide (Blue Origin 2019), currently in Revision F.

\section{Internal Payloads}

As shown in Figure 2, Blue Origin currently offers standard interfaces for in-capsule payloads in four sizes, ranging from cubesat-form factors to large full-stack replacements.

Researchers may also propose custom payload interfaces, ranging from $35 \mathrm{lb}(15.8 \mathrm{~kg})$ for a Single Lockerreplacement to thousands of pounds for a reentry vehicle that could replace the entire capsule. Custom hatches or windows to support unique experiment needs may also be possible.

\section{Standard Interfaces for Payload Lockers}

Blue Origin provides standard Payload Locker hardware in both Single and Double sizes. These lockers are delivered to users in advance of flight to support payload integration and testing.

Locker sides are constructed from sandwich panels of aluminum sheet and aluminum honeycomb core. The front door has a silicone gasket. In addition to a hinged door on the front of each locker, the Payload Locker has a removable top plate for ease of experiment insertion and servicing. The front and rear panels may be replaced for customization, for example, the addition of a vent or fan.

Each payload has access to four switchable DC bus voltage power services (18-36 V, $27 \mathrm{~V}$ nominal), with each service limited to $2 \mathrm{~A}$ max draw by active overcurrent protection circuitry. A payload can therefore access approximately $200 \mathrm{~W}$ peak power.

A flight-tested computer is provided with modules and software to support data acquisition, actuator control, and payload communications. Channels are provided for both analog and digital I/O, RTD sensing, pulsewidth modulation outputs, and Ethernet and RS-232 communications. 32 GB of data storage is provided per Locker.

Vehicle state data are made available in near-real time to the payload via this flight computer over Ethernet. Passed parameters include mission elapsed time, altitude, attitude, 
velocity, acceleration, thruster firings, and mission phases. Payloads may use these signals as triggers in flight or for post-flight correlations. Real-time telemetry to the ground is currently not available.

Power and data services are typically made available during terminal count, approximately 5 min before launch. After a nominal mission, power service is typically maintained until vehicle safing, approximately 5 min later.

\section{External Payloads}

New Shepard's newest payload interface supports external payloads in the vehicle's interstage. Such payloads have direct access to the space environment for testing, atmospheric sampling, TRL-raising, and other in-space pursuits.

Payloads are mounted on the top of the booster, within the vehicle's ring fin. This area is covered at launch, providing a protected ride to the vehicle's nominal staging altitude of approximately $215,000 \mathrm{ft}(65.5 \mathrm{~km})$. At this time in the mission, the main engine cuts off and the capsule is separated from the booster by a spring-loaded system, exposing the interstage fully to the flight environments. The location is in the "ram" position as the propulsion module coasts up to approximately $328,000 \mathrm{ft}(100 \mathrm{~km})$, then becomes the "aft" position as the booster descends to a powered landing back at the launch site. Outboard sensor locations are also available for payloads that need to look out or down.

\section{Concept of Operations}

Blue Origin's West Texas Launch Site, where New Shepard launch operations are performed, is located near Van Horn, Texas.

Blue Origin provides a basic Payload Processing Facility for storage, staging, flight preparations, and post-mission processing of experiments. Payload customers are allotted 2 days in the facility prior to payload handover.

Installation of payloads into the New Shepard capsule will typically occur 4 days prior to launch. Blue Origin personnel will take possession of the Payload Lockers and transfer them to the vehicle for installation. These standard payloads will remain unpowered until launch day and will undergo multiple reorientations during horizontal mating of the crew capsule and propulsion module.

Payloads requiring late access to power, those unable to withstand reorientation, and those necessitating late integration for science purposes may request that Blue Origin install Payload Lockers after the vehicle has been rotated vertically at the pad on the day of launch, approximately $8 \mathrm{~h}$ ahead of launch.

On the day of launch, the mated New Shepard booster and capsule are rolled out horizontally to the launch pad. The vehicle is erected vertically on the launch stand and fueled for flight.
After landing, the capsule and its integrated payloads are craned onto a recovery vehicle and transported back to the Vehicle Processing Facility. Experimental return to the payload customer is expected within $8 \mathrm{~h}$ of crew capsule (CC) landing. If earlier access is required for sample retrieval, special arrangements can be made for access shortly after capsule landing.

\section{FLIGHT ENVIRONMENTS}

\section{Trajectory and Acceleration Profile}

Figure 3 shows altitude above sea level versus time for a $350,000 \mathrm{ft}(107 \mathrm{~km})$ reference trajectory, for both capsule and booster elements.

Sensed accelerations throughout this trajectory result from aerodynamic drag, engine thrust, separation springs, and parachute loads. Maximum ascent loads are typically below $3 \mathrm{G}$, while reentry loads peak around $5 \mathrm{G}$. Transient peak loads on Payload Stacks for a nominal landing are expected as an instantaneous ( $<50 \mathrm{msec}$ ) pulse at $+15 /-4.5 \mathrm{G}$ axial, $\pm 7.5 \mathrm{G}$ lateral.

\section{Microgravity Environment}

Payloads in the CC experience around $3 \mathrm{~min}$ of total microgravity during a typical flight.

The capsule has a system of cold-gas thrusters to perform attitude control during the exo-atmospheric coast phase of flight. On a nominal mission, nearly all thruster firings will happen during the $15 \mathrm{~s}$ immediately following separation from the PM. During this period, the CC reaction control system (RCS) will attempt to null rates in pitch, yaw, and roll axes. After this period, the frequency of RCS thruster firings is expected to drop significantly.

Once maneuvering is complete, the CC enters an extended "Coast Phase" which lasts for an average of $180 \mathrm{~s}$. In-flight accelerometer data collected in collaboration with NASA suggests that the initial period of microgravity after the vehicle has been stabilized can be extremely quiet (Hrovat et al., 2016). During this Coast Phase, the CC achieves uninterrupted microgravity of $<5.0 \times 10^{-3} \mathrm{~g}$ for up to $160 \mathrm{~s}$, with a five-mission average duration of $144 \mathrm{~s}$.

Payloads with sensitivity to high-frequency vibration should consider passive $50 \mathrm{~Hz}$ isolators, appropriately tuned to the mass of the hardware, or an active isolation platform.

\section{Capsule Working Environments}

The pressure inside the $\mathrm{CC}$ is nominally maintained within $10.1-14.2$ psi ( $0.70-0.98$ bar). The air is ambient West Texas composition. The temperature of the cabin air on payload flights is expected to remain in the range of $50-90^{\circ} \mathrm{F}(10$ $32.2^{\circ} \mathrm{C}$ ). Humidity is not actively controlled. 


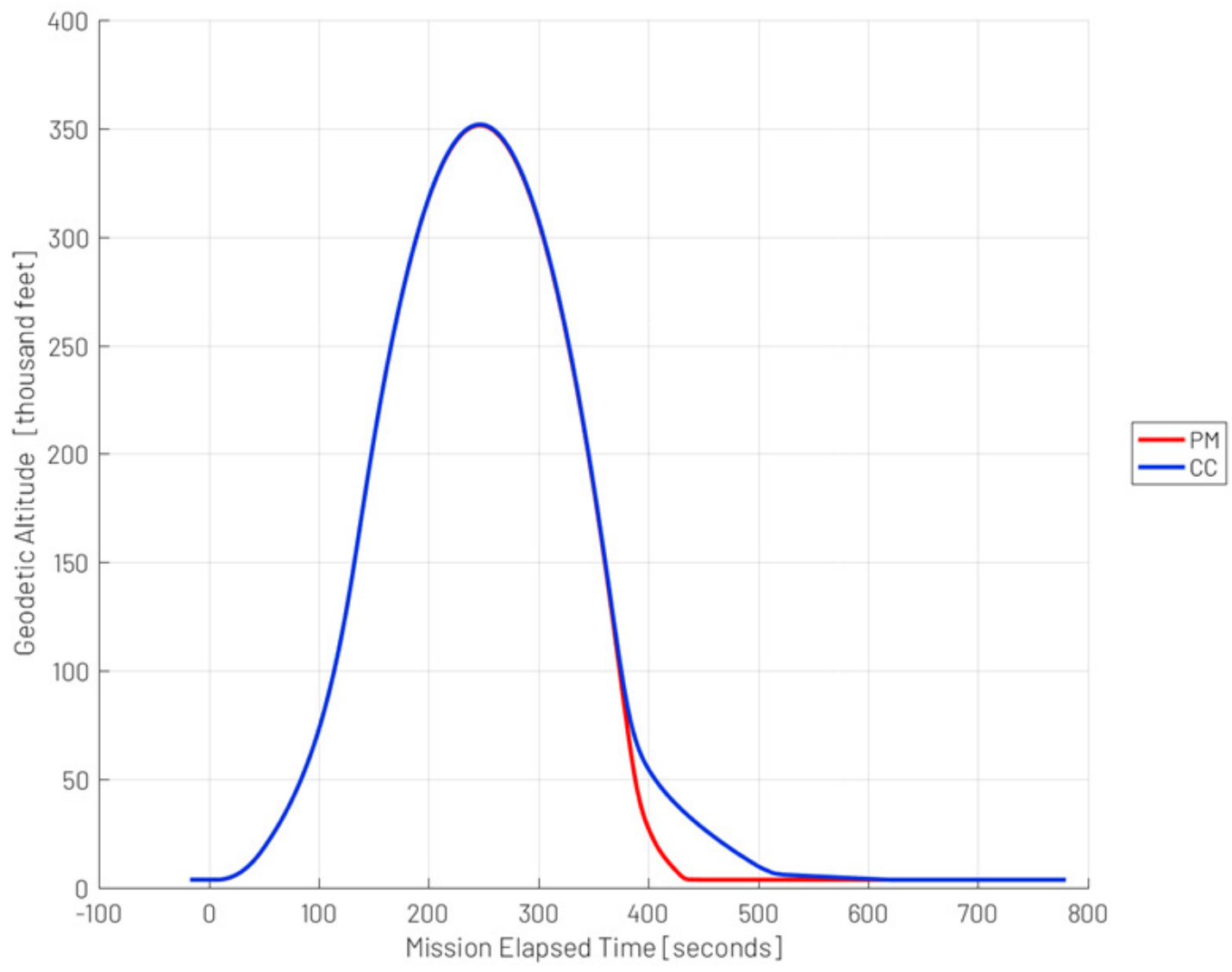

Figure 3. Representative altitude profile for both capsule (CC) and booster (PM) for a 350,000 ft (107 km) reference trajectory (Blue Origin 2019).

Acoustic environments during launch are expected to remain below $125.2 \mathrm{~dB}(\mathrm{~A})$. During the remainder of a nominal flight, acoustic levels are generally considerably lower.

\section{CONCLUSIONS}

Blue Origin's New Shepard vehicle is actively conducting suborbital payload flights, with 10 payload missions and more than 100 payloads having been completed at the time of writing this paper. Flights to space above the Kármán Line provide $3 \mathrm{~min}$ of microgravity exposure, as well as opportunities for high-altitude measurements and demonstrations. A wide variety of experiments in life and physical sciences, Earth and space sciences, technology development, education, and the arts can be supported inside the environmentally controlled capsule and on external mounting. Standard interfaces and streamlined paperwork processes provide ready support for even new payload developers, and human-oriented flight environments simplify hardware design and testing.

\section{REFERENCES}

Blue Origin (2019) New Shepard Payload User's Guide for Research and Education Missions, Rev. F. NSPM-MA0002-F.

Blue Origin (2020). Mini Payload Quick Start Guide - Rev C.

Hrovat K, Pournelle R, Ibrahim A. (2016, 26-28 October) New Shepard Flight Environment. Cleveland, OH, USA: American Society for Gravitational and Space Research. 